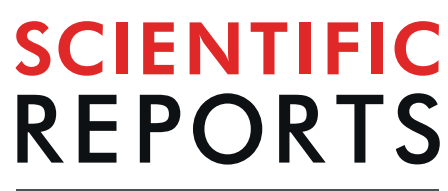

natureresearch

\title{
Vascularized cardiac tissue construction with orientation by layer-by-layer method and 3D printer
}

\author{
Yoshinari Tsukamoto, Takami Akagi \& Mitsuru Akashi* \\ Herein, we report the fabrication of native organ-like three-dimensional (3D) cardiac tissue with an \\ oriented structure and vascular network using a layer-by-layer (LbL), cell accumulation and 3D printing \\ technique for regenerative medicine and pharmaceutical applications. We firstly evaluated the 3D \\ shaping ability of hydroxybutyl chitosan (HBC), a thermoresponsive polymer, by using a robotic \\ dispensing 3D printer. Next, we tried to fabricate orientation-controlled 3D cardiac tissue using \\ human induced pluripotent stem cell-derived cardiomyocytes (hiPSC-CM) and normal human cardiac \\ fibroblasts (NHCF) coated with extracellular matrix (ECM) nanofilms by layer-by-layer technique. \\ These cells were seeded in the fabricated rectangular shape HBC gel frame. After cultivation of the \\ fabricated tissue, fluorescence staining of the cytoskeleton revealed that hiPSC-CM and NHCF were \\ aligned in one direction. Moreover, we were able to measure its contractile behavior using a video \\ image analysis system. These results indicate that orientation-controlled cardiac tissue has more \\ remarkable contractile function than uncontrolled cardiac tissue. Finally, co-culture with human cardiac \\ microvascular endothelial cells (HMVEC) successfully provided a vascular network in orientation- \\ controlled 3D cardiac tissue. The constructed 3D cardiac tissue with an oriented structure and vascular \\ network would be a useful tool for regenerative medicine and pharmaceutical applications.
}

Tissue engineering technology uses living cells, cytokines and biomaterials with the primary goal of creating functional tissue substitutes for novel medical treatments and pharmaceutical development ${ }^{1}$. In the human body, native organs and tissues such as heart, lung, liver and kidney have very complex three-dimensional (3D) structures with highly specialized cellular and extracellular matrix (ECM) components. For example, cardiac tissue has an oriented structure in which cells are aligned in one direction. In the heart, cardiomyocytes have different orientations for each layer, allowing it to produce a large contractile force and stimulus transmission function to deliver blood to the whole body $y^{2,3}$. Therefore, fabrication of 3D tissue with a structure and function similar to native organs for application to medical treatment and pharmaceutical development is required. In order to achieve this, biofabrication technology such as bioprinting and bioassembly for controlling cells and materials is important ${ }^{4}$. Among them, cardiac tissue is one of the important organs for life support ${ }^{5}$. For this reason, human cardiomyocytes (CM) are difficult to use for fabrication of 3D-CM tissues in vitro. Due to recent advances in the development of human induced pluripotent stem cells (hiPSC) and methods to differentiate them to CMs, they have become available as a cell source for the fabrication of $3 \mathrm{D}$ cardiac tissue ${ }^{6,7}$. For the development of novel cell transplantation therapy and drug screening in vivo, it is important to fabricate cardiac tissue with the heart-specific structure of orientation in order to complement organ function.

Many researchers have reported the development of 3D cardiac tissue by using scaffolds such as synthetic substrates and natural macromolecules ${ }^{8-12}$. In addition, other researchers have developed scaffold-free technologies ${ }^{13-15}$, cell sheet engineering ${ }^{16-20}$, and decellularized techniques ${ }^{21-25}$. These methods are high-potency systems of 3D cardiac tissue fabrication. In particular, there have been some reports on the fabrication of orientation-controlled cardiac tissue ${ }^{12,26-30}$. Morimoto et al. have reported a fabrication method of orientation-controlled cardiac tissue by patterning of the hydrogel structures with hiPSC-CM ${ }^{30}$. Moreover, they demonstrated the drug reactivity of fabricated tissue, the changes beating rate and contractile force. Li J et al. 
(a)

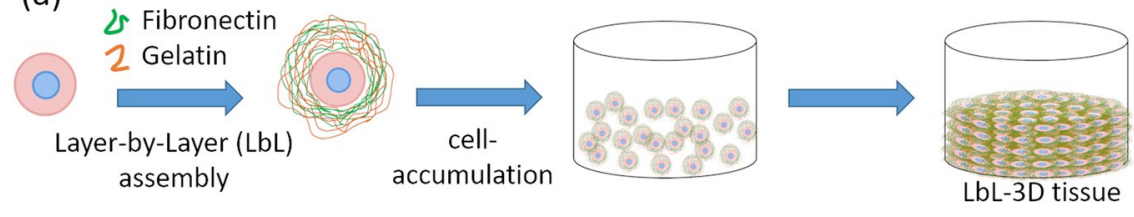

(b)

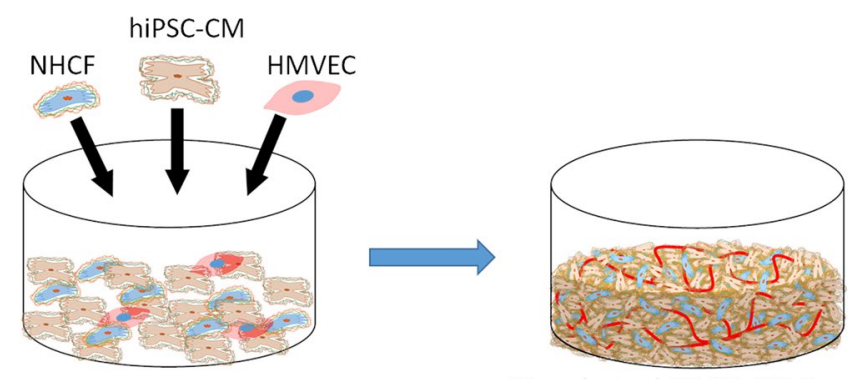

Vascularized 3D iPS-CM tissues

Figure 1. (a) Schematic illustration of the fabrication process for layer-by-layer (LbL) 3D tissue using fibronectin (FN) and gelatin (G) coating technique and cell accumulation technique. (b) Schematic illustration of fabrication of 3D cardiac tissue with a blood capillary network using LbL coated cells and cell accumulation technique.

reported the fabrication of cardiac tissue-like constructs by cultivating hiPSC-CM on aligned nanofibers. They also reported the repair of a myocardial infarction model rat's heart when these tissues were engrafted ${ }^{8}$. On the other hand, cardiac tissues require blood vessels because they are thick and have high cell density thus consuming a large amount of nutrients and oxygen. However, reports about fabrication of 3D cardiac tissue with an oriented and vascularized structure are seldom mentioned and have many problems such as the lack of bottom-up technology. In order to solve this problem, the 3D printer technology which precisely controls the placement of cells and materials is attracting great attention ${ }^{31-36}$.

In our previous study, we developed a fabrication approach of 3D multilayered tissue, "a hierarchical cell manipulation technique", by coating nanometer-sized ECM films of fibronectin (FN) and gelatin (G) onto a cell surface using layer-by-layer (LbL) assembly ${ }^{37}$. In addition, we also developed a rapid bottom-up technique, "cell accumulation technique", by a single cell coating using FN-G nanofilms (Fig. 1a) ${ }^{38}$. We have demonstrated the fabrication of various kinds of 3D tissue models such as skin models ${ }^{39,40}$, blood vessel models ${ }^{41}$, blood/lymph-vascularized cancer metastasis models ${ }^{42}$, pancreas models ${ }^{43}$, liver models $s^{44,45}$ and heart mod$\mathrm{els}^{46}$. Primarily, the heart model was fabricated by using hiPSC-CM and has a vascular network (Fig. 1b). This model was used to evaluate drug response with respect to cardiotoxicity in vitro. Moreover, this vascularized cardiac model was applied to an animal transplantation experiment ${ }^{47}$. However, these models have yet to possess orientation-controlled tissue which is specific to the structure of the heart. It is important to control the orientation of cells for the successful fabrication of functional 3D cardiac tissue models.

Here, we report on a method for the fabrication of 3D cardiac tissue with heart specific structure, cell orientation and vascular network. To achieve this purpose, we reported on a fabrication method of orientation-controlled 3D tissue by using an LbL technique, cell accumulation method and 3D print technology ${ }^{48}$. The cell direction in the $3 \mathrm{D}$ tissue was aligned by controlling the $3 \mathrm{D}$ tissue shape using a 3D printed gel frame. By controlling the shape of the tissue linearly, the cells in the 3D tissue are directionally influenced by patterning and tensile force. In this report, we applied the orientation control method for fabrication of functional 3D cardiac tissue (Fig. 2). At first, we fabricated the gel frame on a culture insert for control cell orientation by using a 3D printer and a thermo-responsive polymer gel, hydroxybutyl chitosan (HBC). $\mathrm{HBC}$ has the ability of sol-gel transition depending on the temperature. In the next step, we fabricated ECM nanofilms onto hiPSC-CM and normal human cardiac fibroblast (NHCF) cells by using the LbL technique. In addition, these cells were seeded in the HBC gel frame by using the cell accumulation method to make orientation-controlled 3D tissue. After culture, cell morphologies and contraction function of the fabricated 3D tissues were evaluated by using fluorescent staining and image analysis ${ }^{49-51}$. Finally, we tried to fabricate native-like $3 \mathrm{D}$ cardiac tissue with orientation and vascular network constructs using co-cultured hiPSC-CM, NHCF and human cardiac microvascular endothelial cells (HMVEC).

\section{Results}

Assessment of $\mathrm{HBC}$ gel printing by robotic dispensing 3D printer. We tried to assess the shaping ability of the robotic dispensing 3D printer for printing an $\mathrm{HBC}$ gel $3 \mathrm{D}$ structure using $\mathrm{HBC}$ as an ink material. The ink cooled to $4^{\circ} \mathrm{C}$ by a Peltier element in the ink tank was printed and assembled into a linear shape on a glass plate heated to $50^{\circ} \mathrm{C}$. The results of this assessment were that the $\mathrm{HBC}$ line width was about $1 \mathrm{~mm}$ and the $\mathrm{HBC}$ gel could be laminated up to 8 layers. Figure 3a shows the measured height of the assembled HBC gel wall. The $\mathrm{HBC}$ gel wall height increased from $300 \mu \mathrm{m}$ to $2 \mathrm{~mm}$ as the lamination number increased from 1 to 8 . Figure $3 \mathrm{~b}$ shows the width of the $\mathrm{HBC}$ gel wall. The $\mathrm{HBC}$ gel wall width increased from $500 \mu \mathrm{m}$ to $1.1 \mathrm{~mm}$. 
(a)

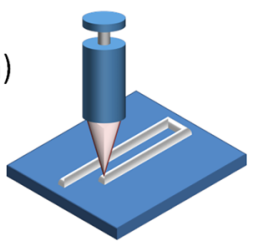

Fabrication of $\mathrm{HBC}$ gel frame by 3D printer

(c)

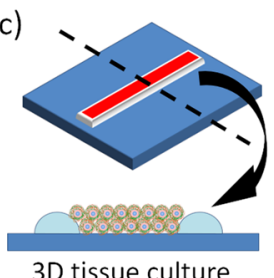

(b)
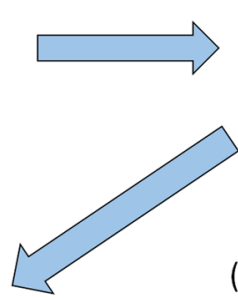

(d)
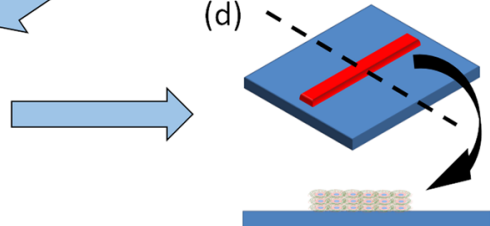

Assessment of

fabricated 3D tissue

Figure 2. Schematic illustration of fabrication of orientation-controlled 3D cardiac tissue using 3D printing technology. (a) 3D printing of HBC using a robotic dispensing 3D printer. (b) Fabrication of 3D multilayer tissue using LbL coated cells and cell accumulation technique. (c) Cultivation of orientation-controlled 3D tissue. (d) Assessment of shape and contractile properties using a histological technique and image processing.

(a)

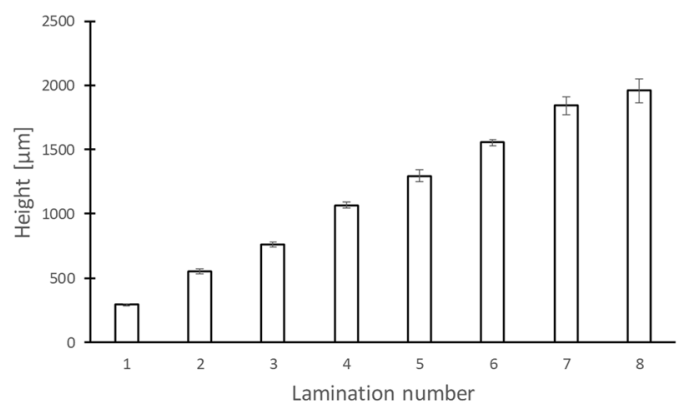

(b)

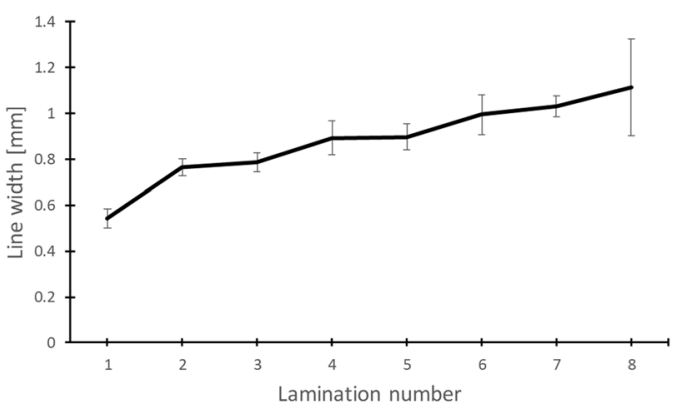

Figure 3. Observation and analysis of a laminated 5\% HBC gel wall printed by a robotic dispensing 3D printer. (a) Height of laminated HBC gel wall observed from the horizontal direction. (b) Line width of laminated HBC gel wall observed from the vertical direction.

Fabrication of orientation-controlled 3D cardiac tissue by HBC polymer gel. We tried to fabricate orientation-controlled 3D cardiac tissue by printed HBC gel frame. In a previous study, we fabricated orientation-controlled 3D tissue of NHCF using a shape controlled rectangular shaped HBC gel frame with a short side of $2 \mathrm{~mm}$ or less ${ }^{48}$. We deduced that orientation can be controlled by limiting the direction of extension and direction of tension by forming the tissue thin. In this study, we focused on the rectangular-shaped HBC gel for fabrication of the orientation-controlled 3D cardiac tissue. The HBC gel was printed onto a culture insert by using the robotic dispensing printer ${ }^{48}$. The fabricated $\mathrm{HBC}$ gel frame shape was rectangular with a long side of $15 \mathrm{~mm}$ and a short side of $1.5 \mathrm{~mm}$. Figure $4 \mathrm{a}-\mathrm{d}$ shows a fabricated shape-controlled 3D cardiac tissue by printed $\mathrm{HBC}$ gel frame with a $1.5 \times 15 \mathrm{~mm}$ rectangle (The overview image was shown in Supplementary Fig. 1). Figure $4 \mathrm{e}-\mathrm{h}$ shows an uncontrolled $3 \mathrm{D}$ cardiac tissue by culture in a 24 -well insert. Figures $4 \mathrm{a}-\mathrm{h}$ show the fluorescent staining image of fabricated 3D cardiac tissue. Figure 4c,g show cTnT, a known cardiac marker, in fabricated 3D cardiac tissue stained with Alexa 546 conjugated antibody. Figure 4d,h show F-actin of NHCF and cardiomyocytes in fabricated 3D cardiac tissue stained with Acti-stain 488 fluorescent phalloidin (See Supplementary Fig. 2). Figure 4i,j show the assessment result of the cell orientation obtained from F-actin images by ImageJ software. Cell orientation was calculated from the F-actin fiber angle of NHCF and cardiomyocytes. These graphs show 3D cardiac tissue by HBC gel frame with a $1.5 \times 15 \mathrm{~mm}$ rectangle (Fig. 4i) and 24-well culture insert (Fig. 4j). From these data, the number of cells oriented in the same direction in the 3D cardiac tissue by $\mathrm{HBC}$ gel frame with a $1.5 \times 15 \mathrm{~mm}$ rectangle was higher than the $3 \mathrm{D}$ cardiac tissue in 24 -well culture insert.

Contractile properties of fabricated 3D cardiac tissues. Next, we tried to assess the contractile properties of fabricated 3D cardiac tissues using an image analysis technique. Figure 5a,b show the result of the motion vector images calculated from phase contrast images of orientation-controlled 3D cardiac tissue (Fig. 5a) and uncontrolled 3D cardiac tissue (Fig. 5b) (The original movie is shown as Supplementary Movies 1 and 2). The arrows' direction and color indicate the contraction direction and the contraction speed of the $3 \mathrm{D}$ cardiac tissues. 

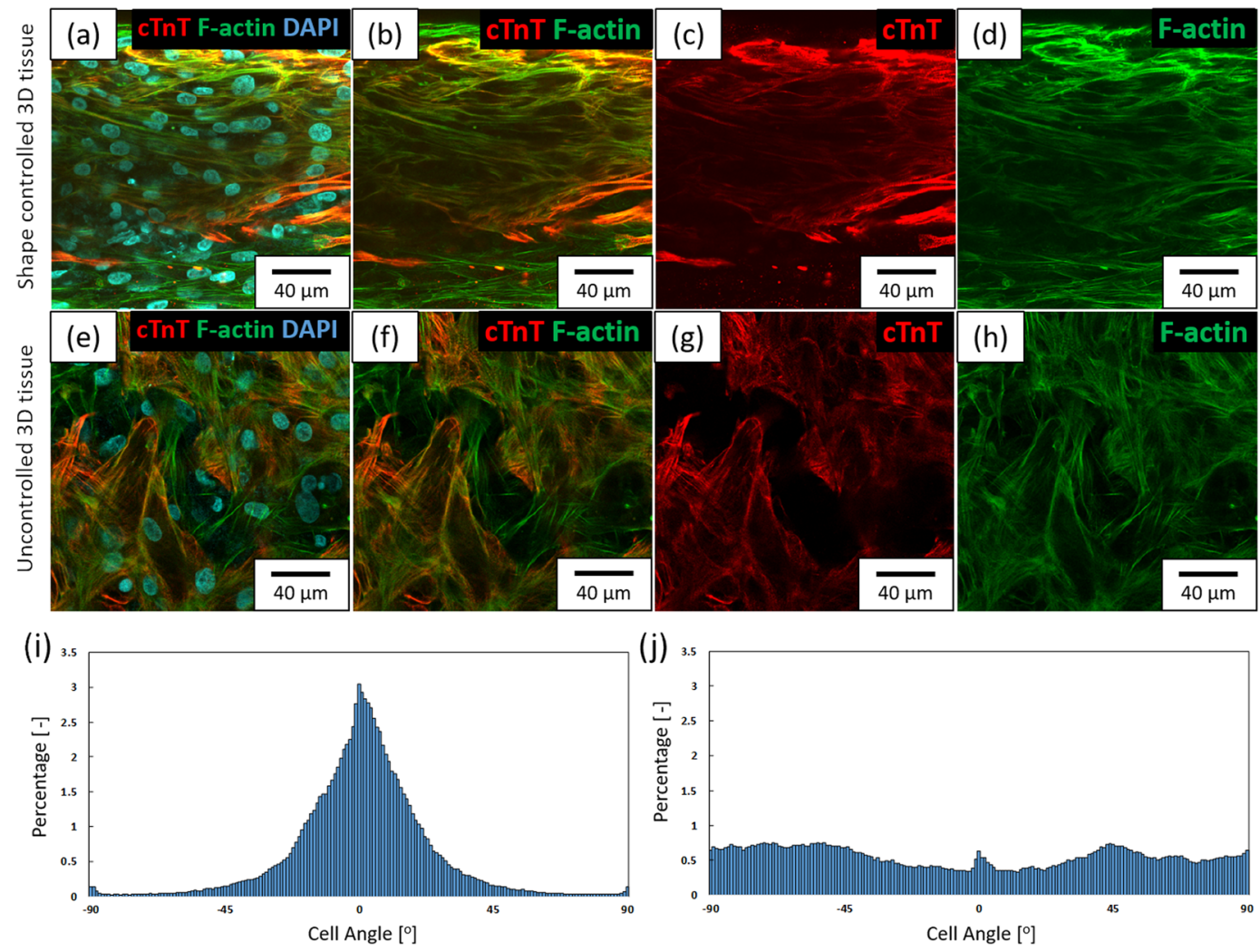

Figure 4. Shape controlled 3D cardiac tissue image stained with fluorescent labeling phalloidin and anticardiac troponin T (cTnT) antibody obtained from confocal laser scanning microscopy (CLSM). (a-d) Shape controlled 3D cardiac tissue using a $2 \times 15 \mathrm{~mm} \mathrm{HBC}$ gel frame. (e-h) Uncontrolled 3D cardiac tissue. (a,e) The merged images of F-actin, cTnT and DAPI. (b,f) The merged images of F-actin and cTnT. (c,g) The cTnT images. $(\mathbf{d}, \mathbf{h})$ The F-actin images. (i,j) The graphs of the local alignment angles of F-actin fibers in shape controlled 3D cardiac tissue are shown underneath the CLSM image by image analysis.

From these results, the cardiomyocytes of orientation-controlled 3D tissue moved in the same direction and synchronized a large number of cells. In the case of uncontrolled 3D cardiac tissue, cardiomyocytes moved in different directions and the contracting area was smaller than in the orientation-controlled 3D cardiac tissue. Figure $5 \mathrm{c}$ shows the average velocity and time intervals of orientation-controlled $3 \mathrm{D}$ cardiac tissues (blue) and uncontrolled 3D cardiac tissues (orange). From these data, it was established that the contraction velocity of orientation-controlled 3D cardiac tissue was faster than uncontrolled tissues. Figure 5d shows the difference between orientation-controlled tissue and uncontrolled tissue in maximum contraction speed and relaxation speed. From these data it was established that orientation-controlled tissue contracted and relaxed twice as quickly as uncontrolled tissue.

Fabrication of the orientation-controlled 3D cardiac tissue with vascular network. Finally, we constructed orientation-controlled 3D cardiac tissues with vascular network. To introduce the vascular network into the 3D cardiac tissue, hiPSC-CMs and NHCF coated FN-G nanofilms by LbL technique were co-cultured with HMVEC in a $1.5 \times 15 \mathrm{~mm}$ rectangular HBC gel frame (5\%). After 5 days of culture, hiPSC-CM and HMVEC in the fabricated 3D cardiac tissues were fixed and stained by immunofluorescence stain. Figure 6b,e show cTnT images of hiPSC-CM stained with anti-cTnT antibody to investigate the distribution of hiPSC-CM in 3D cardiac tissue. Figure $6 \mathrm{c}$, f show CD31 images of HMVEC stained with anti-CD3l, a specific endothelial marker stained to investigate the shape and distribution of the vascular network. Figure $6 \mathrm{a}-\mathrm{c}$ show a fabricated shape-controlled $3 \mathrm{D}$ cardiac tissue by printed $\mathrm{HBC}$ gel frame with a $1.5 \times 15 \mathrm{~mm}$ rectangle. Figure $6 \mathrm{~d}-\mathrm{f}$ show an uncontrolled $3 \mathrm{D}$ cardiac tissue by culture in a 24 -well insert. As a result of hiPSC-CM, NHCF and HMVEC co-culture, the orientation of hiPSC-CM could be controlled by using a $1.5 \mathrm{~mm}$ short side rectangular HBC gel frame. Figure $6 \mathrm{~g}, \mathrm{~h}$ show the assessment result of the vascular network orientation obtained from CD31 images by ImageJ software. Cell orientation was calculated from vascular network angle of HMVEC. From these data, vascular network has orientation structure in the orientation-controlled $3 \mathrm{D}$ cardiac tissue, but not has orientation structure in the uncontrolled 3D cardiac tissue. 


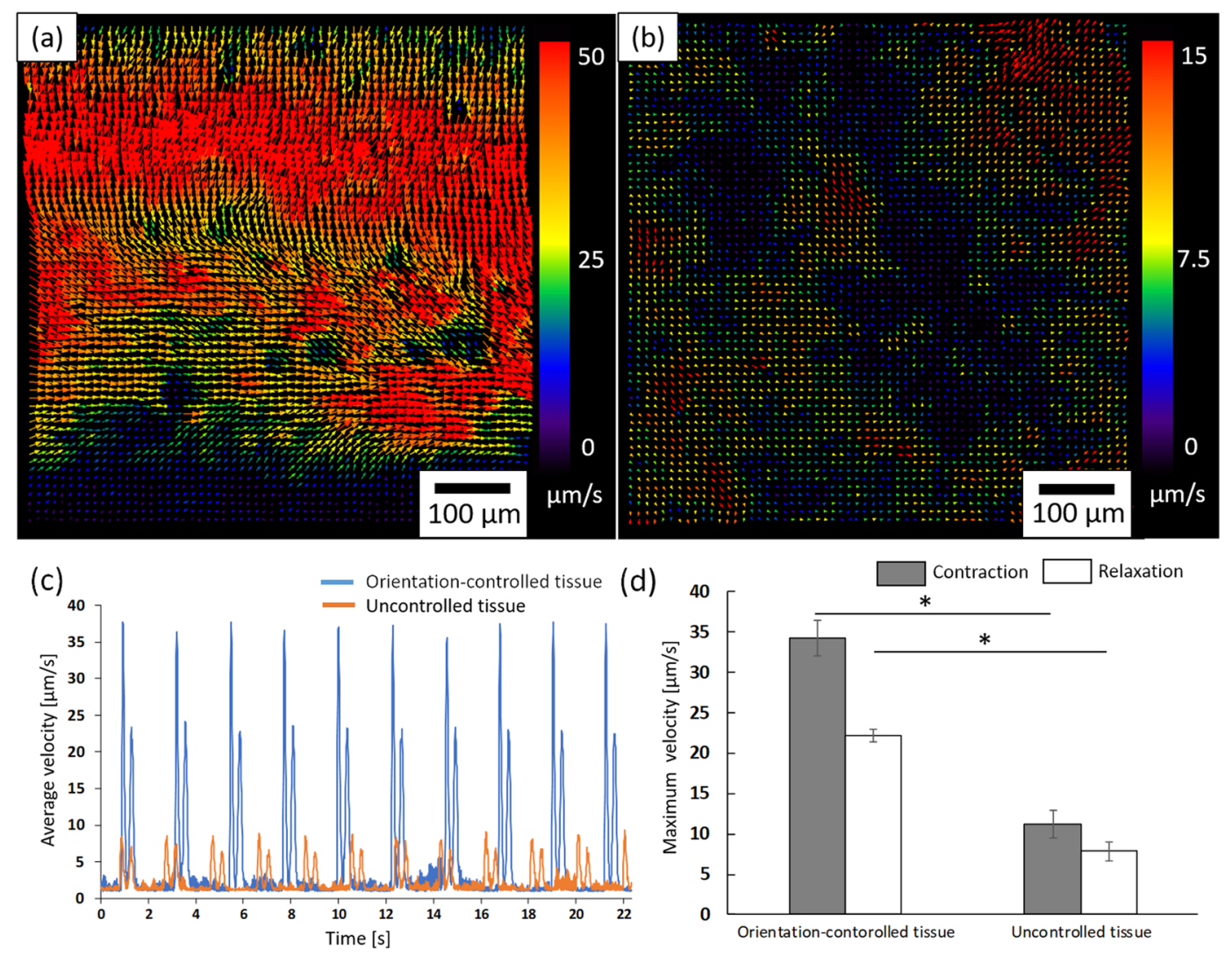

Figure 5. The motion analyses of surface movement of $3 \mathrm{D}$ cardiac tissue calculated by particle image velocimetry (PIV). (a) The motion analysis result of orientation-controlled 3D cardiac tissue. (b) The motion analysis result of orientation uncontrolled tissue. The arrows indicate the direction of movement of the separated $21 \mu \mathrm{m}$ square block and the colors show the velocity of the movement. The color scales are as indicated beside each of the figures. (c) Plot of a motion waveform representing contraction and relaxation peaks. The blue plot indicates the orientation-controlled tissue. The orange plot indicates the uncontrolled tissue. (d) The graph of maximum contraction and relaxation velocity. $(* p<0.05, \mathrm{n}=3)$.

\section{Discussion}

Artificial 3D tissues are in great demand in regenerative medicine and the drug development field for medical treatments and in vitro assays. In the fabrication of artificial 3D tissues, it is necessary to regulate the microenvironment such as placement of multiple cells and ECM. This is because the organs and tissues in the human body appeared to have multiple functions and specific structures such as 3D structure, cell-ECM interaction and cell-cell interaction. We have reported a fabrication method of 3D tissue with only cells and ECM using an LbL technique and cell accumulation technique. This technique is able to control the cell type per layer and construct blood capillaries in the 3D tissue. It has the advantages of high cell density for cell-cell interaction and early vascular network formation compared with other methods of 3D tissue fabrication, such as using porous scaffolds, nanofiber scaffolds and hydrogels. The fabrication of biomimetic 3D tissue with a multilayered structure like skin was easily achieved using an LbL and cell accumulation technique. However, the reproduction of complex structures such as orientation of muscle cells and polarity of hepatocytes was difficult by this technique. In our previous study, we could fabricate the 3D cardiac tissue by hiPSC-CM and use it for drug evaluation. In addition, we reported a fabrication method of orientation-controlled 3D tissue by $3 \mathrm{D}$ printing technology ${ }^{48}$. The cells in a linearly controlled 3D tissue are aligned in one direction by the influence of patterning and tensile force. As known, cells recognize the surrounding environment such as topography and tensile force from other cells. In the case of 3D tissue fabrication, cells are able to have an oriented structure by recognizing topography and tensile force like 2D culture. In this study, we tried to fabricate orientation-controlled 3D cardiac tissue by using an LbL technique, cell accumulation technique and 3D printing technology.

At first, we assessed the capability of 3D modeling by using a robotic dispensing 3D printer and $\mathrm{HBC}$ gel. The $\mathrm{HBC}$ gel could be assembled to at least 8 layers with a linear shape (Fig. 3a). A ninth layer could not be laminated because the HBC gel wall melted. The reason for this is that the ninth layer is far from the substrate and melts because it cannot receive temperature control. From our previous studies, however, the thickness of $3 \mathrm{D}$ tissue is limited to $100 \mu \mathrm{m}$. For this reason, the 3D modeling ability of HBC gel is sufficient to fabricate 3D tissue using an LbL technique and cell accumulation technique. In addition, HBC gel has the ability of sol-gel transition in response to temperature. For this reason, only the $\mathrm{HBC}$ gel can be removed by cooling and only the fabricated 3D tissue can be collect. 

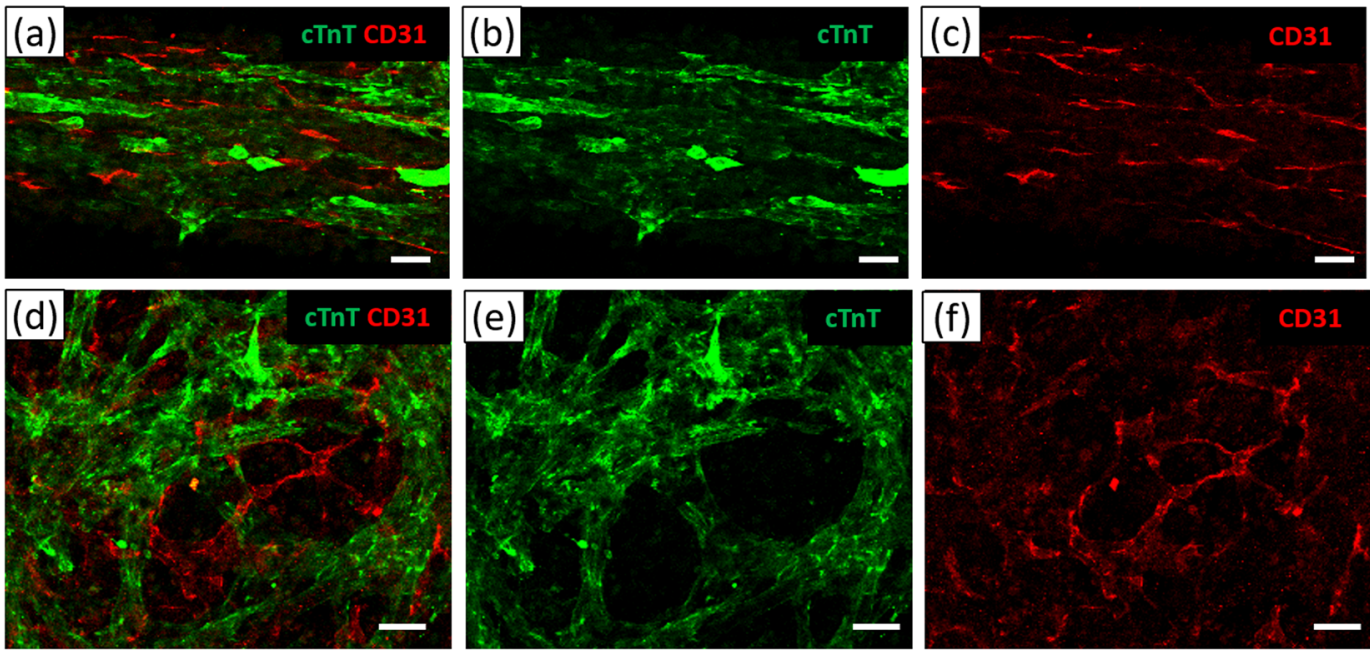

Scale bar: $50 \mu \mathrm{m}$
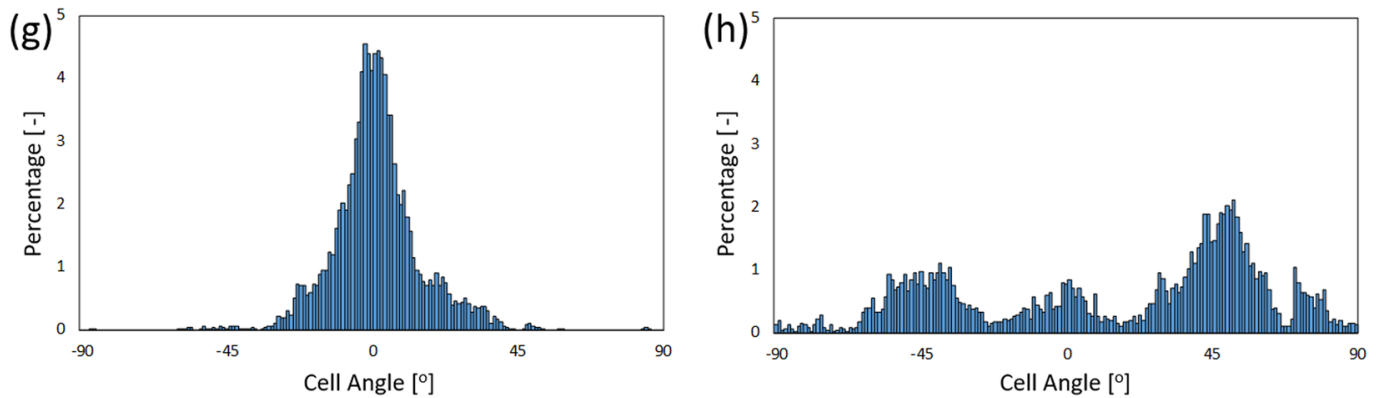

Figure 6. Shape controlled 3D cardiac tissue with vascular network image stained anti-cardiac troponin $\mathrm{T}$ (cTnT) antibody and anti-CD31 antibody obtained from LSCM. (a-c) Shape controlled 3D cardiac tissue using a $1.5 \times 15 \mathrm{~mm}$ HBC gel frame. (d,e,f) Uncontrolled 3D cardiac tissue. (a,d) The merged image of cTnT (green) and CD31 (red). (b,e) The cTnT image. (c,f) The CD31 image. (g,h) The graphs of the local alignment angles of vascular network (CD31) in shape controlled 3D cardiac tissue are shown underneath the CLSM image by image analysis. (g) The graph of orientation-controlled tissue. (h) The graph of uncontrolled tissue.

Next, we fabricated orientation-controlled 3D cardiac tissue by using 3D printing of HBC gel, LbL technique and cell accumulation technique. In our previous study, we were able to fabricate orientation-controlled 3D fibroblast tissue using these techniques ${ }^{48}$. When the 3D tissue shape was controlled by the printed HBC gel frame with a $2 \times 5 \mathrm{~mm}$ rectangle shape, the cells in the $3 \mathrm{D}$ tissue extended in one direction. Based on this result, we tried to fabricate orientation-controlled 3D cardiac tissue. We observed the morphologies of hiPSC-CM and NHCF stained with F- actin and cTnT by fluorescence dye in fabricated 3D cardiac tissue (Fig. 4a-h). The assessment result of the cell orientation obtained from F-actin images (Fig. 4i,j) indicated that the cell orientation could e controlled in the case of cardiomyocytes by using a printed HBC gel frame with a $1.5 \times 15 \mathrm{~mm}$ rectangle. Comparing the fabricated 3D cardiac tissue with the native heart tissue, the cell density in native heart tissue is $10^{8-9} \mathrm{cells} / \mathrm{cm}^{352,53}$. On the other hand, the cell density of the $3 \mathrm{D}$ cardiac tissue fabricated by the tissue fabrication method by the LbL technique used in this study was about $6 \times 10^{8}$ cells $/ \mathrm{cm}^{3}$ calculated. Native heart tissue has the orientation structure and different orientation in each layer ${ }^{54}$. On the other hand, although the 3D cardiac tissue fabricated by LbL technique can not be achieved yet, it has a unidirectionally oriented structure. This result is similar to our previous study and indicates that these techniques are suitable methods for fabrication of orientation-controlled 3D cardiac tissues.

Moreover, we tried to assess the contractile properties of fabricated 3D cardiac tissues by image analysis. Figure 5a,b indicate that orientation-controlled 3D cardiac tissue contracted in one direction compared to uncontrolled tissue. In addition, Fig. 5c shows that the contraction speed of orientation-controlled 3D cardiac tissue was faster than the uncontrolled one. Figure $5 \mathrm{~d}$ indicates the quantitative value of maximum contraction and relaxation speed obtained from a plot of the average velocity and time intervals. From this quantitative value, orientation-controlled tissue contracts more than twice as quickly as uncontrolled tissue. In the heart of the living body, the orientation of cardiomyocytes is known to be an important factor for producing a large contractile force and stimulus transmission function ${ }^{2,3}$. For these reasons, this assessment result indicates that it is possible to fabricate cardiac tissue with a function similar to a living body using the techniques outlined in this study.

Finally, we tried to fabricate orientation-controlled 3D cardiac tissue with a vascular network by using an HBC gel frame with a $1.5 \times 15 \mathrm{~mm}$ rectangle. Vascular network is important structures in the fabrication of $3 \mathrm{D}$ tissue for use in regenerative medicine and drug development fields which require thicker tissue. The reason for the necessity of blood vessels is that oxygen diffusion is limited to a depth of about $100 \mu \mathrm{m}$ from the tissue surface . $^{55}$. 
If there are no blood vessels, the inner cells of 3D tissues that are thicker than $100 \mu \mathrm{m}$ become necrotic due to lack of oxygen ${ }^{56}$. For this reason, we developed a fabrication method for 3D cardiac tissue with vascular network. Figure $6 \mathrm{a}-\mathrm{f}$ show the hiPSC-CM stained cTnT and HMVEC stained CD31 immunofluorescence staining images obtained from orientation-controlled cardiac tissue (Fig. 6a-c) and uncontrolled cardiac tissue (Fig. 6d-f). From the result of CD31 stained images, vascular network formed in both tissues. In the case of orientation-controlled tissue, the vascular network has an oriented structure similar to cardiomyocytes according to image analysis (Fig. 6g). In the case of uncontrolled tissue, on the other hand, the vascular network does not have an oriented structure (Fig. 6h). Rosenfield et al. reported on the orientation of vascular network in a 3D gel structure ${ }^{57}$. They indicated that the orientation of vascular network was formed by tensile force in the 3D tissue. In our study, we consider that vascular network orientation in the fabricated 3D cardiac tissue was caused by tensile force which regulated the orientation in one direction. However, in order to fabricate thicker and functional tissue, perfusion culture utilizing vascular network is required. Therefore, we work on tissue fabrication using perfusion culture. The obtained data suggest that orientation-controlled 3D cardiac tissue with vascular network would be efficacious in transplantation therapy and drug assessment.

We revealed that orientation-controlled tissue made by this study has native organ like structure and high function. We are conducting research to apply this tissue as a more effective therapeutic effect than conventional tissue. Moreover, since it is expected to exhibit reaction similar to native organ, we are working on application as a drug discovery model. Additionally, it is expected to be applied to tissues having oriented structures such as cardiac tissue as well as skeletal muscle, nerve and vascular tissue. From the above, this research is considered to contribute greatly to regenerative medicine and drug discovery research.

In conclusion, we successfully fabricated 3D cardiac tissues with an oriented structure and vascular network using an LbL technique, cell accumulation technique and 3D printing technology using a thermoresponsive polymer gel as an ink material. The fabricated orientation-controlled 3D cardiac tissue had an oriented structure and exhibited better contractile properties than the uncontrolled tissue. The controlled tissue contracted in one direction and showed a high speed of contraction. Moreover, the orientation-controlled 3D cardiac tissue has a vascular network. This $3 \mathrm{D}$ cardiac tissue has the potential for usage in transplantation medical care and drug assessment because it has the native heart organ-like structure and vascular network for the fabrication of thicker and larger 3D tissue. Therefore, we believe that the 3D cardiac tissue with orientation and vascular network would be a useful tool for regenerative medicine and pharmaceutical applications.

\section{Materials and Methods}

Materials. All of the chemical reagents were used without further purification. Chitosan was kindly donated by Dainichiseika Color \& Chemicals Mfg. Co. Ltd (Tokyo, Japan). Fibronectin from human plasma, Triton X-100, human recombinant FGF-2, 1-thioglycerol and bovine serum albumin were purchased from Sigma-Aldrich (MO, USA). Gelatin, L-ascorbic acid 2-phosphate trisodium salt, IWR-1-endo and Y-27632 were purchased from Wako Pure Chemical Industries (Osaka, Japan). fetal bovine serum, 4'6-diamidino-2-phenylindole, dihydrochloride (DAPI), Alexa Fluor 546-conjugated goat anti-rabbit or anti-mouse IgG cross-absorbed secondary antibody, Alexa Fluor 488-conjugated goat anti-mouse IgG cross-absorbed secondary antibody, Knockout-DMEM/F12, knockout serum replacement, 2-mercaptoethanol, MEM non-essential amino acids, L-glutamine and StemPro-34 were purchased from Thermo Fisher Scientific (MA, USA). Cell culture inserts with a $0.4 \mu \mathrm{m}$ pore membrane (polyester) and cell culture inserts with a $3 \mu \mathrm{m}$ pore membrane (polycarbonate) were purchased from Corning (NY, USA). NHCF, HMVEC, fibroblast growth medium (FGM-3), and endothelial growth medium (EGM-2 MV) were purchased from Lonza (Basel, Switzerland). Acti-stain 488 fluorescent phalloidin and Acti-stain 555 fluorescent phalloidin were purchased from Cytoskeleton, Inc (CO, USA). Rabbit polyclonal anti-CD31 antibody and anti-cardiac troponin $\mathrm{T}$ (cTnT) antibody were purchased from Abcam (Cambridge, UK). The monoclonal mouse anti-human CD31 antibody was purchased from Dako (Glostrup, Denmark) (CA, USA). Dulbecco's modified eagle medium (DMEM), Antibiotic-antimycotic mixed stock solution and 4\% paraformaldehyde (PFA)/PBS were purchased from Nacalai Tesque (Kyoto, Japan). 1,2-butylene oxide (BO) was purchased from Tokyo Chemical Industry Co., Ltd (Tokyo, Japan). Human iPS cell line 253G1 was obtained from RIKEN Bio Resource Center (Ibaraki, Japan). Mitomycin C-inactivated mouse embryo fibroblast feeder cells, dispase II and VEGF165 were purchased from Millipore Co. (MA, USA), Roche Diagnostics (Basel, Switzerland) and HumanZyme (IL, USA), respectively. Recombinant human proteins of BMP-4 and activin A were purchased from R\&D systems Inc (MN, USA). Accutase and Accumax ware purchased from Innovative Cell Technologies, Inc (CA, USA). IWP-2 was purchased from TOCRIS Bioscience (Bristol, UK).

Synthesis of thermoresponsive polymer. We used $\mathrm{HBC}$ gel that has a thermoresponsive function as an ink material for the 3D printer. HBC gel is known to have the ability of sol-gel transition in response to temperature. In this study, we synthesized HBC gel using a previously reported method ${ }^{58}$. Chitosan polymer was dissolved into $0.1 \mathrm{M}$ hydrogen chloride $(\mathrm{HCl})$. Sodium hydroxide $(\mathrm{NaOH})(5 \mathrm{M})$ was added into the chitosan solution to adjust the $\mathrm{pH}$ to 8 . After warming the chitosan solution to $85^{\circ} \mathrm{C}, \mathrm{BO}$ was added and it was mixed for $3 \mathrm{~h}$. Some sediment of $\mathrm{HBC}$ appeared during the reaction. The sediment was dissolved with $5 \mathrm{M} \mathrm{HCl}$. Next, another $\mathrm{BO}$ was added and the reaction was continued at $85^{\circ} \mathrm{C}$ for $24 \mathrm{~h}$. The product was then purified by dialysis in pure water at room temperature for $24 \mathrm{~h}$ and the purified product was collected by lyophilization for 4 days. The degree of substitution (DS) of the polymer was determined by elemental analysis referring to a previous report ${ }^{59}$.

The DS was computed by the following equation: $\mathrm{DS}=\left\{(\mathrm{C} / \mathrm{N})_{\mathrm{m}}-(\mathrm{C} / \mathrm{N})_{0}\right\} / 4$, where $(\mathrm{C} / \mathrm{N})_{\mathrm{m}}$ indicated $\mathrm{C} / \mathrm{N}$ (mole ratios) of the chitosan derivative, $(\mathrm{C} / \mathrm{N})_{0}$ indicated the $\mathrm{C} / \mathrm{N}$ (mole ratios) of the chitosan ${ }^{59}$.

Cell preparation. Human iPS cells $253 \mathrm{G} 1^{60}$ ware cultured on a Mitomycin C-treaded mouse embryonic fibroblast feeder layer in KnockOut Serum Replacement (KSR) based medium supplemented with 4 ng/ml FGF-2. 
KSR-based medium consisted of knockout-DMEM/F12 medium, supplemented with 20\% (v/v) KSR, $0.1 \mathrm{mM}$ 2-mercaptoethanol, MEM non-essential amino acids, and $2 \mathrm{mM}$ L-glutamine. hiPSC were passaged every 7 days as small clumps by treatment with $1 \mathrm{mg} / \mathrm{ml}$ dispase II, followed by pipetting.

For hiPSC-CM differentiation, hiPSC clumps after accutase treatment, pipetting and $100 \mu \mathrm{m}$ cell strainer (Corning, NY, USA) were suspended in $30 \mathrm{ml}$ StemPro-34 medium containing $50 \mu \mathrm{g} / \mathrm{ml} \mathrm{L}$-ascorbic acid 2-phosphate trisodium salt, $2 \mathrm{mM}$ L-glutamine and $400 \mu \mathrm{M}$ 1-thioglycerol, and induce differentiation added $10 \mu \mathrm{M}$ Y-27632, $10 \mathrm{ng} / \mathrm{ml}$ BMP-4, $5 \mathrm{ng} / \mathrm{ml} \mathrm{FGF-2,} 3 \mathrm{ng} / \mathrm{ml}$ activin A. Cell suspension were seeded into a $30 \mathrm{ml}$ single-use bioreactor (ABLE Corporation \& Biott Co., Japan) at $37^{\circ} \mathrm{C}$, an agitation rate of $55 \mathrm{rpm}$. The culture medium was exchange containing: $10 \mathrm{ng} / \mathrm{ml} \mathrm{BMP}-4,5 \mathrm{ng} / \mathrm{ml} \mathrm{FGF-2} \mathrm{and} 3 \mathrm{ng} / \mathrm{ml}$ activin A on day 2; $4 \mu \mathrm{M}$ IWR1 and $10 \mu \mathrm{M}$ IWP-2 on day 3 and day $5 ; 5 \mathrm{ng} / \mathrm{ml}$ VEGF and $10 \mathrm{ng} / \mathrm{ml} \mathrm{FGF-} 2$ on day 6, 8, 10 and $12^{61}$.

On day 13, hiPSC-CM were dissociated spheroids into single cell suspensions by Accumax treatment and disruption through repeated pipetting and $40 \mu \mathrm{m}$ cell strainer (Corning, NY, USA). The single cells of hiPSC-CM were suspended in DMEM with 10\% FBS. The cTnT positive rate of the used cardiomyocytes was more than $90 \%$. In addition, in this study, cardiomyocytes were used without purification ${ }^{61}$.

NHCF (passage was less than 7) were cultured with FGM-3 and HMVEC (passage was less than 7) were cultured with EGM-2 $\mathrm{MV}$ at $37^{\circ} \mathrm{C}$ in an incubator at $5 \% \mathrm{CO}_{2}$.

Designing 3D frames with a thermoresponsive polymer using a 3D printer. We used a dispenser type 3D printer (SHOTMASTER 200DS; Musashi Engineering) for printing the thermoresponsive polymer and fabricating the 3D structure (See Supplementary Fig. 3 and Supplementary Movie 3). Figure 2a shows a schematic of the robotic dispenser type 3D printer. This printer has a dispensing nozzle consisting of a $1 \mathrm{~mL}$ syringe with a double thread screw taper nozzle with an internal diameter of $610 \mu \mathrm{m}$. In addition, this printer has other modules such as a syringe pump module, $\mathrm{Y}$ moving stage and the $\mathrm{X}-\mathrm{Z}$ moving motors module and temperature control module with a Peltier element. By using the temperature control module, the ink tank was cooled to $4^{\circ} \mathrm{C}$ and the stage was heated up to $50^{\circ} \mathrm{C}$. The HBC $(50 \mathrm{mg} / \mathrm{mL})$ solution was loaded into the ink tank. In this study, the syringe pump, moving speed, and nozzle-to-collector distance were set at $7 \mu \mathrm{L} / \mathrm{s}, 2.5 \mathrm{~mm} / \mathrm{s}$, and $250 \mu \mathrm{m} / \mathrm{layer}$ respectively. After the HBC gels print, we assessed the shape of the printed HBC gels by observing them from a horizontal angle and measuring the laminated HBC gel height by ImageJ software ${ }^{62}$. We prepared the 6-well culture insert with a $0.4 \mu \mathrm{m}$ pore membrane and the HBC gel was printed onto the membrane (nozzle cooled to $4^{\circ} \mathrm{C}$, stage heated up to $50^{\circ} \mathrm{C}$ ). Finally, we fabricated the $\mathrm{HBC}$ gel with a rectangular shape where the long side was $15 \mathrm{~mm}$ and the short side was $2 \mathrm{~mm}$ and $3 \mathrm{~mm}$ frame onto 6 well culture insert.

Fabrication of ECM nanofilm using a filtration-LbL method. Isolated hiPSC-CM and NHCF were coated with FN-G nanofilms by filtration LbL method according to our previous report ${ }^{46}$. For the filtration-LbL, we prepared $2.5 \mathrm{~mL}$ of $0.2 \mathrm{mg} / \mathrm{mL}$ FN in PBS and G in PBS solution and PBS were added into 3 wells in a 6-well plate. Isolated hiPSC-CM and NHCF were suspended in $500 \mu \mathrm{L}$ of PBS after centrifugation and added to a 6-well culture insert with a $3 \mu \mathrm{m}$ pore membrane. The insert was immersed in the FN solution and agitated at $500 \mathrm{rpm}$ for $1 \mathrm{~min}$ at room temperature using a MixMate shaker (Eppendorf). After FN coating, the cells were washed with PBS. Next, the cells were immersed in the G solution and agitated again under the same conditions as FN. After the G coat, the cells were washed with PBS. These steps were considered to be one cycle and four cycles were completed. Finally, the cells were coated with an FN coat. (FN: five times, G: four times).

Fabrication of orientation-controlled 3D cardiac tissues using a cell accumulation technique. After the LbL coating process, FN-G nanofilms were formed on each cell membrane. The $5 \mathrm{~mL}$ cell suspension mixed with LbL coated hiPSC-CM $\left(5.4 \times 10^{6}\right.$ cells $)$ and NHCF $\left(1.8 \times 10^{6}\right.$ cells $)$ were seeded into the 6-well culture inserts which were set in a 6-well plate. An HBC gel frame coated with fibronectin and $2 \mathrm{~mL}$ culture medium was added to the outside of the culture insert. After $2 \mathrm{~h}$ culture, $5 \mathrm{~mL}$ medium was added into the well to connect the inner and outer of the insert medium in the culture insert. It was then incubated in $5 \% \mathrm{CO}_{2}$ at $37^{\circ} \mathrm{C}$. In the case of fabrication of $3 \mathrm{D}$ cardiac tissue with blood capillaries, $5 \mathrm{~mL}$ cell suspension mixed with ECM nanofilm-coated hiPSC-CM $\left(5.4 \times 10^{6}\right.$ cells $)$ and NHCF $\left(1.8 \times 10^{6}\right.$ cells $)$ and uncoated HMVEC $\left(7.1 \times 10^{5}\right.$ cells $)$ were seeded into the 6-well culture inserts which were set in a 6-well plate. An HBC gel frame coated with fibronectin and $2 \mathrm{~mL}$ culture medium was added to the outside of the culture insert. After $2 \mathrm{~h}$ culture, $5 \mathrm{~mL}$ medium was added into the well to connect the inner and outer of the insert medium in the culture insert. It was then incubated in $5 \% \mathrm{CO}_{2}$ at $37^{\circ} \mathrm{C}$. To compare cell morphology with shape controlled $3 \mathrm{D}$ cardiac tissue, uncontrolled $3 \mathrm{D}$ cardiac tissue was fabricated by using 24 -well culture insert. The $400 \mu \mathrm{L}$ cell suspension mixed with LbL coated hiPSC-CM $\left(0.75 \times 10^{6}\right.$ cells $)$ and NHCF $\left(0.25 \times 10^{6}\right.$ cells $)$ were seeded into the 24 -well culture inserts which were set in a 24 -well plate. In the case of $3 \mathrm{D}$ cardiac tissue with vascular network, the $400 \mu \mathrm{L}$ cell suspension mixed with LbL coated hiPSC-CM $\left(0.75 \times 10^{6}\right.$ cells $)$, NHCF $\left(0.25 \times 10^{6}\right.$ cells $)$ and HMVEC $\left(1.0 \times 10^{5}\right.$ cells $)$ were seeded into the 24-well culture inserts which were set in a 24-well plate. Add $1 \mathrm{~mL}$ culture medium to the outside of the culture insert. After $2 \mathrm{~h}$ culture, $1 \mathrm{~mL}$ medium was added into the well to connect the inner and outer of the insert medium in the culture insert. It was then incubated in $5 \% \mathrm{CO}_{2}$ at $37^{\circ} \mathrm{C}$.

Fluorescence staining of 3D cardiac tissues and microscopy. For the evaluation of the 3D cardiac tissues, the samples were washed with PBS, fixed with $4 \%$ PFA and permeabilized with $0.2 \%$ Triton X for 20 min at room temperature. After washing with PBS, samples were blocked with $1 \%$ bovine serum albumin (BSA) for 1 hour. The blocked samples were incubated with primary antibodies at $4{ }^{\circ} \mathrm{C}$ overnight. The samples were subsequently incubated with secondary antibodies at room temperature for 2 hours. F-actin was stained with Acti-stain 488 fluorescent for $60 \mathrm{~min}$ at room temperature in darkness. cTnT was stained with monoclonal anti-cTnT antibody (1:300) as the primary antibody and Alexa 546 conjugated anti-mouse IgG antibody (1:200) or Alexa 488 
conjugated anti-mouse IgG antibody (1:200) as the secondary antibody. CD31 staining, was used polyclonal anti-CD31 antibody (1:200) as the primary antibody and Alexa 546 conjugated anti-rabbit IgG antibody (1:200) as the secondary antibody. Following this, samples washed with PBS and stained the cell nuclei with DAPI (10 $\mu \mathrm{g} /$ $\mathrm{mL}$ ) for $30 \mathrm{~min}$ at room temperature in darkness. The stained samples were observed by confocal laser scanning microscopy (CLSM) (FLUOVIEW FV10i: Olympus) (LSM 780: Carl Zeiss).

Evaluation of cardiac tissue cell orientation and contractile properties. ImageJ plug-in, OrientationJ, was used for the quantification of the fabricated 3D tissue's cell orientation ${ }^{63,64}$. This plug-in showed the directional distribution of the actin fibers and vascular networks from observed fluorescence staining images. Moreover, we tried to assess the contractile properties of the fabricated 3D cardiac tissue by image analysis with the ImageJ plug-in, particle image velocimetry (PIV $)^{62,65}$. This method calculates the cross correlations between two consecutive images divided into small blocks and shows the velocity and direction of contraction.

Statistical analysis. The contractile properties of orientation-controlled 3D cardiac tissue $(n=3)$ and uncontrolled $3 \mathrm{D}$ cardiac tissue $(\mathrm{n}=3)$ were evaluated from the PIV analysis. These data were expressed as means \pm standard deviation (SD). Comparisons between each tissue contraction velocity and relaxation velocity were performed by a Student's t-test. A $p$ value less than 0.05 was considered be statistically significant.

Received: 26 February 2019; Accepted: 6 November 2019;

Published online: 26 March 2020

\section{References}

1. Langer, R. \& Vacanti, J. P. Tissue engineering. Science 260, 920-926 (1993).

2. LeGrice, I. J. et al. Laminar structure of the heart: ventricular myocyte arrangement and connective tissue architecture in the dog. Am. J. Physiol. 269, H571-H582 (1995).

3. Costa, K. D., Takayama, Y., McCulloch, A. D. \& Covell, J. W. Laminar fiber architecture and three-dimensional systolic mechanics in canine ventricular myocardium. Am. J. Physiol. 276, H595-H607 (1999).

4. Groll, J. et al. Biofabrication: reappraising the definition of an evolving field. Biofabrication 8, 013001 (2016).

5. Chen, Q. Z., Harding, S. E., Ali, N. N., Lyon, A. R. \& Boccaccini, A. R. Biomaterials in cardiac tissue engineering: ten years of research survey. Mater. Sci. Eng. 59, 1-37 (2008).

6. Takahashi, K. et al. Induction of pluripotent stem cells from adult human fibroblasts by defined factors. Cell 131, 861-872 (2007).

7. Ralphe, J. C. \& de Lange, W. J. 3D engineered cardiac tissue models of human heart disease: Learning more from our mice. Trends. Cardiovasc. Med. 23, 27-32 (2013).

8. Li, J. et al. Human pluripotent stem cell-derived cardiac tissue-like constructs for repairing the infarcted myocardium. Stem Cell Reports 9, 1546-1559 (2017).

9. Nakane, T. et al. Impact of cell composition and geometry on human induced pluripotent stem cells-derived engineered cardiac tissue. Sci. Rep. 7, 45641 (2017).

10. Eschenhagen, T. et al. Three-dimensional reconstitution of embryonic cardiomyocytes in a collagen matrix: a new heart muscle model system. FASEB J. 11, 683-694 (1997).

11. Zimmermann, W. H. et al. Three-dimensional engineered heart tissue from neonatal rat cardiac myocytes. Biotechnol. Bioeng. 68, 106-114 (2000).

12. Zhang, D. et al. Tissue-engineered cardiac patch for advanced functional maturation of human ESC-derived cardiomyocytes. Biomaterials 34, 5813-5820 (2013).

13. Desroches, B. R. et al. Functional scaffold-free 3-D cardiac microtissues: a novel model for the investigation of heart cells. Am. J. Physiol. Heart. Circ. Physiol. 302, H2031-H2042 (2012).

14. Vukusis, K. et al. High Density sphere culture of adult cardiac cells increases the levels of cardiac and progenitor markers and shows signs of vasculogenesis. BioMed. Res. Int. 2013, 696837 (2013).

15. Ravenscroft, S. M., Pointon, A., Williams, A. W., Cross, M. J. \& Sidaway, J. E. Cardiac non-myocyte cells show enhanced pharmacological function suggestive of contractile maturity in stem cell derived cardiomyocyte microtissues. Toxicol. Sci. 152, 99-112 (2016).

16. Yamato, M. \& Okano, T. Cell sheet engineering. Mater. today 7, 42-47 (2004).

17. Miyagawa, S. et al. Tissue cardiomyoplasty using bioengineered contractile cardiomyocyte sheets to repair damaged myocardium: Their integration with recipient myocardium. Transplantation 80, 1586-1595 (2005).

18. Matsuura, K. et al. Creation of human cardiac cell sheets using pluripotent stem cells. Biochem. Biophys. Res. Commun. 425, 321-327 (2012).

19. Haraguchi, Y., Shimizu, T., Yamato, M. \& Okano, T. Regenerative therapies using cell sheet-based tissue engineering for cardiac disease. Cardiol. Res. Pract. 2011, 845170 (2011).

20. Shimizu, T. et al. Fabrication of pulsatile cardiac tissue grafts using a novel 3-Dimensional cell sheet manipulation technique and temperature-responsive cell culture surfaces. Circ. Res. 90, e40-e48 (2002).

21. Ott, H. C. et al. Perfusion-decellularized matrix: using nature's platform to engineer a bioartificial heart. Nat. Med. 14, 213-221 (2008).

22. Lu, T. Y. et al. Repopulation of decellularized mouse heart with human induced pluripotent stem cell-derived cardiovascular progenitor cells. Nat. Commun. 4, 2307 (2013).

23. Yasui, H. et al. Excitation propagation in three-dimensional engineered hearts using decellularized extracellular matrix. Biomaterials 35, 7839-7850 (2014)

24. Russo, V., Omidi, E., Samani, A., Hamilton, A. \& Flynn, L. E. Porous, ventricular extracellular matrix-derived foams as a platform for cardiac cell culture. Biores Open Access 4, 374-388 (2015).

25. Williams, C., Quinn, K. P., Georgakoudi, I. \& Black, L. D. III. Young developmental age cardiac extracellular matrix promotes the expansion of neonatal cardiomyocytes in vitro. Acta Biomater. 10, 194-204 (2014).

26. Baudino, T. A. et al. Cell patterning: interaction of cardiac myocytes and fibroblasts in three-dimensional culture. Microsc Microanal 14, 117-125 (2008)

27. Salick, M. R. et al. Micropattern width dependent sarcomere development in human ESC-derived cardiomyocytes. Biomaterials 35, 4454-4464 (2014).

28. Cimetta, E. et al. Production of arrays of cardiac and skeletal muscle myofibers by micropatterning techniques on a soft substrate. Biomed. Microdevices. 11, 389-400 (2009).

29. Bian, W., Liau, B., Badie, N. \& Bursac, N. Mesoscopic hydrogel molding to control the 3D geometry of bioartificial muscle tissues. Nat. Protoc. 4, 1522-1534 (2009). 
30. Morimoto, Y., Mori, S., Sakai, F. \& Takeuchi, S. Human induced pluripotent stem cell-derived fiber-shaped cardiac tissue on a chip. Lab. Chip. 16, 2295-2301 (2016).

31. Calvert, P. Printing cells. Science 318, 208-209 (2007).

32. Arai, K. et al. Three-dimensional inkjet biofabrication based on designed images. Biofabrication 3, 034113 (2011).

33. Arai, K. et al. The development of cell-adhesive hydrogel for 3D printing. Int. J. Bioprinting 2, 44-53 (2016).

34. Arai, K. et al. Fabrication of 3D-culture platform with sandwich architecture for preserving liver-specific functions of hepatocytes using 3D bioprinter. J. Biomed. Mater. Res. A 105, 1583-1592 (2017).

35. Sakai, S., Ueda, K., Gantumur, E., Taya, M. \& Nakamura, M. Drop-On-Drop Multimaterial 3D Bioprinting Realized by PeroxidaseMediated Cross-Linking. Macromol. Rapid. Commun. 39, 1700534 (2018).

36. Xu, T., Baicu, C., Aho, M., Zile, M. \& Boland, T. Fabrication and characterization of bio-engineered cardiac pseudo tissues. Biofabrication 1, 035001 (2009).

37. Matsusaki, M., Kadowaki, K., Nakahara, Y. \& Akashi, M. Fabrication of cellular multilayers with nanometer-sized extracellular matrix films. Angew. Chem. Int. Ed. Engl. 46, 4689-4692 (2007).

38. Nishiguchi, A., Yoshida, H., Matsusaki, M. \& Akashi, M. Rapid construction of three-dimensional multilayered tissues with endothelial tube networks by the cell-accumulation technique. Adv. Mater. 23, 3506-3510 (2011).

39. Matsusaki, M. et al. Development of full-thickness human skin equivalents with blood and lymph-like capillary networks by cell coating technology. J. Biomed. Mater. Res. A 103, 3386-3396 (2015).

40. Akagi, T., Nagura, M., Hiura, A., Kojima, H. \& Akashi, M. Construction of three-dimensional dermo-epidermal skin equivalents using cell coating technology and their utilization as alternative skin for permeation studies and skin irritation tests. Tissue Eng. Part A 23, 481-490 (2017).

41. Nishiguchi, A., Matsusaki, M., Asano, Y., Shimoda, H. \& Akashi, M. Effects of angiogenic factors and 3D-microenvironments on vascularization within sandwich cultures. Biomaterials 35, 4739-4748 (2014).

42. Nishiguchi, A. et al. In vitro 3D blood/lymph-vascularized human stromal tissues for preclinical assays of cancer metastasis. Biomaterials 179, 144-155 (2018).

43. Fukuda, Y. et al. Layer-by-layer cell coating technique using extracellular matrix facilitates rapid fabrication and function of pancreatic $\beta$-cell spheroids. Biomaterials 160, 82-91 (2018).

44. Sasaki, K. et al. Construction of three-dimensional vascularized functional human liver tissue using a layer-by-layer cell coating technique. Biomaterials 133, 263-274 (2017).

45. Matsusaki, M., Sakaue, K., Kadowaki, K. \& Akashi, M. Three-dimensional human tissue chips fabricated by rapid and automatic inkjet cell printing. Adv. Healthc. Mater. 2, 534-539 (2013).

46. Amano, Y. et al. Development of vascularized iPSC derived 3D-cardiomyocyte tissues by filtration Layer-by-Layer technique and their application for pharmaceutical assays. Acta Biomater. 33, 110-121 (2016).

47. Narita, H. et al. Engraftment and morphological development of vascularized human iPS cell-derived 3D cardiomyocyte tissue after xenotransplantation. Sci. Rep. 7, 13708 (2017).

48. Tsukamoto, Y., Akagi, T., Shima, F. \& Akashi, M. Fabrication of orientation-controlled 3D tissues using a Layer-by-Layer technique and 3D printed a thermoresponsive gel frame. Tissue Eng. Part C Methods 6, 357-366 (2017).

49. Hayakawa, T. et al. Noninvasive evaluation of contractile behavior of cardiomyocyte monolayers based on motion vector analysis. Tissue Eng. Part C Methods 18, 21-32 (2012).

50. Hayakawa, T. et al. Image-based evaluation of contraction-relaxation kinetics of human-induced pluripotent stem cell-derived cardiomyocytes: Correlation and complementarity with extracellular electrophysiology. J. Mol. Cell Cardiol. 77, 178-191 (2014).

51. Takeda, M. et al. Development of in vitro drug-induced cardiotoxicity assay by using three-dimensional cardiac tissues derived from human induced pluripotent stem cells. Tissue Eng. Part C Methods 24, 56-67 (2018).

52. Mandarim-De-Lacerda, C. A. \& Meirelles Pereira, L. M. Numerical density of cardiomyocytes in chronic nitric oxide synthesis inhibition. Pathobiology 68, 36-42 (2000).

53. Serpooshan, V. et al. Bioacoustic-enabled patterning of human iPSC-derived cardiomyocytes into 3D cardiac tissue. Biomaterials 131, 47-57 (2017).

54. Bensley, J. G., De Matteo, R., Harding, R. \& Black, M. J. Three-dimensional direct measurement of cardiomyocyte volume, nuclearity, and ploidy in thick histological sections. Sci. Rep. 6, 23756 (2016).

55. Tsai, A. G., Johnson, P. C. \& Intaglietta, M. Oxygen gradients in the microcirculation. Physiol. Rev. 83, 933-963 (2003).

56. Anada, T., Fukuda, J., Sai, Y. \& Suzuki, O. An oxygen-permeable spheroid culture system for the prevention of central hypoxia and necrosis of spheroids. Biomaterials 33, 8430-8441 (2012).

57. Rosenfeld, D. et al. Morphogenesis of 3D vascular networks is regulated by tensile forces. Proc. Natl. Acad. Sci. USA 113, 3215-3220 (2016).

58. Kato, A., Kan, K., Ajiro, H. \& Akashi, M. Development of a rapid in vitro tissue deadhesion system using the thermoresponsive solgel transition of hydroxybutyl chitosan. J. Biomater. Sci. Polym. Ed. 28, 958-973 (2017).

59. Wang, Q. Q. et al. Hydroxybutyl chitosan thermo-sensitive hydrogel: a potential drug delivery system. J. Mater. Sci. 48, 5614-5623 (2013).

60. Nakagawa, M. et al. Generation of induced pluripotent stem cells without Myc from mouse and human fibroblasts. Nat. Biotechnol. 26, 101-106 (2008).

61. Sasano, Y., Fukumoto, K., Tsukamoto, Y., Akagi, T. \& Akashi, M. Construction of 3D cardiac tissue with synchronous powerful beating using human cardiomyocytes from human iPS cells prepared by a convenient differentiation method. Journal of Bioscience and Bioengineering (2020).

62. Rasband, W. S. \& ImageJ, U. S. National Institutes of Health, Bethesda, Maryland, USA, http://rsb.info.nih.gov/ij/, 1997-2012.

63. Rezakhaniha, R. et al. Experimental investigation of collagen waviness and orientation in the arterial adventitia using confocal laser scanning microscopy. Biomech. Model Mechanobiol. 11, 461-473 (2012).

64. Fonck, E. et al. Effect of aging on elastin functionality in human cerebral arteries. Stroke 40, 2552-2556 (2009).

65. Tseng, Q. et al. Spatial organization of the extracellular matrix regulates cell-cell junction positioning. Proc. Natil. Acad. Sci. USA 109, 1506-1511 (2012).

\section{Acknowledgements}

This work was supported by "Development of Manufacturing Technology for Functional Tissues and Organs Employing Three-Dimensional Biofabrication” founded by the Japan Agency for Medical Research and Development (AMED) (P14028), Japan Science and Technology Agency (JST) (13A1204), and Grant-in-Aid for JSPS Research Fellow $(30 \bullet 151)$. We thank Y. Sasano (Nagase \& CO., LTD.) and K. Fukumoto (Kyowa Hakko Bio Co., LTD.) for technical support about hiPSC-CM culturing.

\section{Author contributions}

Y.T., T.A. and M.A. designed this study. Y.T. conducted the experiments and acquired data. Y.T., T.A. and M.A. mainly wrote the manuscript. M.A. supervised this study. All authors approved the manuscript. 


\section{Competing interests}

The authors declare no competing interests.

\section{Additional information}

Supplementary information is available for this paper at https://doi.org/10.1038/s41598-020-59371-y.

Correspondence and requests for materials should be addressed to M.A.

Reprints and permissions information is available at www.nature.com/reprints.

Publisher's note Springer Nature remains neutral with regard to jurisdictional claims in published maps and institutional affiliations.

(c) (1) Open Access This article is licensed under a Creative Commons Attribution 4.0 International License, which permits use, sharing, adaptation, distribution and reproduction in any medium or format, as long as you give appropriate credit to the original author(s) and the source, provide a link to the Creative Commons license, and indicate if changes were made. The images or other third party material in this article are included in the article's Creative Commons license, unless indicated otherwise in a credit line to the material. If material is not included in the article's Creative Commons license and your intended use is not permitted by statutory regulation or exceeds the permitted use, you will need to obtain permission directly from the copyright holder. To view a copy of this license, visit http://creativecommons.org/licenses/by/4.0/.

(C) The Author(s) 2020 